\title{
Conductive metallic filaments dominate in hybrid perovskite-based memory devices
}

\author{
Yang Huang ${ }^{1,3}$, Zhenxuan Zhao ${ }^{2^{*}}$, Chen Wang ${ }^{1^{*}}$, Hongbo Fan ${ }^{1}$, Yiming Yang ${ }^{1}$, Jiming Bian ${ }^{3}$ and \\ Huaqiang $\mathrm{Wu}^{2^{*}}$
}

\begin{abstract}
Organic-inorganic hybrid perovskites (OHPs) are well-known as light-absorbing materials in solar cells and have recently attracted considerable attention for the applications in resistive switching memory. Previous studies have shown that ions can migrate to form a conductive channel in perovskites under an external voltage. However, the exact resistance mechanism for Ag or halogens which dominate the resistive behavior is still controversial. Here, we demonstrate a resistive switching memory device based on $\mathrm{Ag} / \mathrm{FA}_{0.83} \mathrm{MA}_{0.17} \mathrm{~Pb}\left(\mathrm{I}_{0.82} \mathrm{Br}_{0.18}\right)_{3} /$ fluorine doped tin oxide (FTO). The migration of Ag cations and halide anions is demonstrated by energy dispersive $\mathrm{X}$-ray spectroscopy (EDS) after the SET process (positive voltage on $\mathrm{Ag}$ ). By comparing the $I-V$ behavior of the Au-based devices, it is clear that the conductive channel formed by $\mathrm{Ag}$ is the main factor of the switching characteristics for Ag-based devices. Meanwhile, by controlling the appropriate SET voltage, two kinds of resistance characteristics of the analog switch and threshold switch can be realized in the Ag-based device. As a result, it may be possible to implement both data storage and neuromorphic computing in a single device.
\end{abstract}

Keywords: Ag filament, perovskite memory, analog switch, threshold switch, resistance mechanism

\section{INTRODUCTION}

Organic-inorganic hybrid perovskite (OHP) materials possess a high absorption coefficient and mobility $[1,2]$, low trap-state density $[3,4]$, small exciton binding energy [5] and a long carrier diffusion [3]. Several OHP-based devices, including photovoltaics [6], light-emitting diodes [7], photodetectors [8], and triboelectric nanogenerators [9], with high performance have recently been assembled.
Many studies suggest that $\mathrm{I}^{-}$and $\mathrm{Br}^{-}$can mobilize and induce electric fields because of their low migration activation energies $[10,11]$ and shallow defects. When an external voltage is applied to the OHP films with a bidirectional scan, a hysteresis characteristic makes OHPs become promising materials for applications in memory devices $[12,13]$.

The first resistive random access memory (ReRAM) device based on OHPs was reported by Yoo et al. [14] in 2015. These OHPs show excellent resistive switching behaviors in terms of high endurance, retention time, and ON/OFF ratios. The structural flexibility and low temperature processing make OHPs a promising candidate for manufacturing wearable devices [15]. In addition, ReRAM devices can be used not only in data storage but also in the field of artificial synapses [16,17]. However, high efficiency resistive memories were rarely reported, and the ionic mobility and atomic diffusion coefficient that form the conductive channel in memory devices still need additional exploration.

The general formula for OHPs is $\mathrm{ABX}_{3}$, where $\mathrm{A}$ is a monovalent cation such as methyl-ammonium (MA), formamidinium (FA), or cesium; $\mathrm{B}$ is a divalent metal cation such as lead or tin; and $\mathrm{X}$ is a halide anion. The crystal structure supplies the lattice with sufficient room to accommodate different cations and anions. Therefore, OHP materials are not limited to the common $\mathrm{CH}_{3} \mathrm{NH}_{3}$ $\mathrm{PbI}_{3}$ form to crystallize. It has been reported that the stability and efficiency can be improved by incorporating $\mathrm{FA}^{+}$with $\mathrm{MA}^{+}$at the A site, along with a mixture of $\mathrm{Cl}$, $\mathrm{Br}$, and $\mathrm{I}$ halide anions at the $\mathrm{X}$ site $[18,19]$. It is apparent that the composition in the $\mathrm{A}$ and/or $\mathrm{X}$ sites alters the device functionality by tuning its crystal structure or film

\footnotetext{
${ }^{1}$ School of Microelectronics, Dalian University of Technology, Dalian 116024, China

${ }^{2}$ Institute of Microelectronics, Tsinghua University, Beijing 100084, China

${ }^{3}$ Key Laboratory of Materials Modification by Laser, Ion and Electron Beams (Ministry of Education), School of Physics, Dalian University of Technology, Dalian 116024, China

* Corresponding authors (emails: wchen@dlut.edu.cn (Wang C); zhaozhenx@163.com (Zhao Z); wuhq@tsinghua.edu.cn (Wu H))
} 
morphology. Several studies have shown that composition engineering of OHPs can alter the light/field-induced ionic migration $[11,13]$. For example, incorporating $\mathrm{MA}^{+}$ into $\mathrm{FAPbI}_{3}$ weakens the Gibbs free energy due to lattice contraction and an increasing cation disorder [20]. In addition, the mixing $\mathrm{FA}^{+}$and $\mathrm{MA}^{+}$at the $\mathrm{A}$ site can enhance the thermal stability [18]. This characteristic can form conductive pathways that are induced by the electric field. While there are several reports related to high efficiency of solar cells based on $\mathrm{FA}_{x} \mathrm{MA}_{1-x} \mathrm{PbX}_{3}$ [21-23], there are few reports regarding its resistance behavior and its application in memory devices.

In this work, we studied $\mathrm{FA}_{0.83} \mathrm{MA}_{0.17} \mathrm{~Pb}\left(\mathrm{I}_{0.82} \mathrm{Br}_{0.18}\right)_{3}$ thin films with a low roughness in a memory device in which $\mathrm{Ag}$ and fluorine doped tin oxide (FTO) were the upper and lower electrodes, respectively. Furthermore, we analyzed changes in the $\mathrm{Ag}, \mathrm{I}$, and $\mathrm{Pb}$ contents before and after exposure to electric fields using the energy dispersive $\mathrm{X}$-ray spectroscopy (EDS), and a clear Ag migration was observed. Furthermore, by comparing the $I-V$ characteristics of the Au-based device, it is clear that the conductive channel formed by Ag is the main factor causing the switching behavior of Ag-based devices. Meanwhile, by controlling the appropriate SET voltage, two kinds of resistance characteristics of analog switches and threshold switches can be realized in Ag-based devices.

\section{EXPERIMENTAL SECTION}

\section{Materials}

$\mathrm{PbI}_{2}$ (98.0\%) and $\mathrm{PbBr}_{2}$ (98.0\%) (TCI), formamidinium iodide (FAI 99.5\%) and methyl-ammonium bromide (MABr 99.5\%) (Xi'an Polymer Light Technology Corp), acetone (99.5\%, Beijing Shiji), ethanol (99.7\%), and isopropanol alcohol (IPA 99.7\% Sinopharm Chemical Reagent Co., Ltd) were used as received. Dimethylformamide (DMF, 99.8\%), dimethylsulfoxide (DMSO, 99.9\%), and chlorobenzene (99.8\%) were purchased from Sigma Aldrich and used without further purification.

\section{Perovskite precursor solution preparation}

$\mathrm{FA}_{0.83} \mathrm{MA}_{0.17} \mathrm{~Pb}\left(\mathrm{I}_{0.82} \mathrm{Br}_{0.18}\right)_{3}$ was prepared with an anhydrous mixture of DMF and DMSO (volume ratio of 4:1) containing FAI $\left(1.0 \mathrm{~mol} \mathrm{~L}^{-1}\right), \mathrm{PbI}_{2}\left(1.1 \mathrm{~mol} \mathrm{~L}^{-1}\right), \mathrm{MABr}$ $\left(0.2 \mathrm{~mol} \mathrm{~L}^{-1}\right)$, and $\mathrm{PbBr}_{2}\left(0.22 \mathrm{~mol} \mathrm{~L}^{-1}\right)$ by stirring at $50^{\circ} \mathrm{C}$ for $1 \mathrm{~h}$. It should be noted that the added volume of $\mathrm{Pb}^{2+}$ and $\mathrm{I}^{-}$ions was designed much more than their stoichiometric ratio of $(\mathrm{A}: \mathrm{B}: \mathrm{X}=1: 1: 3)$ for perovskite $\left(\mathrm{ABX}_{3}\right.$, $\left.\mathrm{A}=\mathrm{FA}^{+} / \mathrm{MA}^{+}, \mathrm{B}=\mathrm{Pb}^{2+}, \mathrm{X}=\mathrm{Cl}^{-} / \mathrm{Br}^{-}\right)$and the excess amount of $\mathrm{Pb}^{2+}$ and $\mathrm{I}^{-}$is 0.12 and $0.24 \mathrm{~mol} \mathrm{~L}^{-1}$, respectively.

\section{Resistive switching memory device fabrication}

Firstly, FTO glasses were sequentially washed with deionized water, ethanol, acetone, and isopropanol. Secondly, the washed FTO glasses were treated with UVozone for $30 \mathrm{~min}$. Then, the remaining film preparation was carried out in a glove box filled with dry air. Afterwards, $30 \mu \mathrm{L}$ of the perovskite precursor solution was dropped on the FTO substrates followed by a two-stage spin coating process. The first spin coating stage was at $1,000 \mathrm{rpm}$ for $10 \mathrm{~s}$ with a $1 \mathrm{~s}$ acceleration. The second spin coating stage was at $6,000 \mathrm{rpm}$ for $20 \mathrm{~s}$ with a $6 \mathrm{~s}$ acceleration in which $150 \mu \mathrm{L}$ of chlorobenzene was dropped on the spinning substrate at $5 \mathrm{~s}$ prior to the end of the program to speed up the crystallization. Then, the substrates were annealed at $100^{\circ} \mathrm{C}$ for $50 \mathrm{~min}$ for good crystallization. Finally, $250 \mathrm{~nm} \mathrm{Ag} / \mathrm{Au}$ electrodes were thermally evaporated under a high vacuum using a mask with the holes of $200 \mu \mathrm{m}$ in diameter.

\section{Characterization}

Scanning electron microscopy (SEM) and EDS were performed with a FEI FEG450. The atomic force microscopy (AFM) images were captured using a Bruker Dimension Icon FastScan system. The crystal structure of the $\mathrm{FA}_{0.83} \mathrm{MA}_{0.17} \mathrm{~Pb}\left(\mathrm{I}_{0.82} \mathrm{Br}_{0.18}\right)_{3}$ films was examined in the $\theta-2 \theta$ mode with X-ray diffraction (XRD, Bruker D8 ADVANCE) using $\mathrm{Cu}$ Ka radiation $(\lambda=0.1542 \mathrm{~nm})$. The electrical properties of the devices were characterized using a Keithley 4200 semiconductor analyzer under ambient and dark conditions. Steady-state photoluminescence (PL) was analyzed by Edinburgh LP 920 Laser Flash spectrometer with an excitation at $404 \mathrm{~nm}$. Time-resolved photoluminescence (TRPL) was obtained by using the time-correlated single photon counter technology. Excitation of the sample was achieved with a pulse laser (PIXEA-CU-1, AUREA, France) of $406 \mathrm{~nm}$ wavelength, $0.5 \mathrm{MHz}$ repetition rate and $\sim 35 \mathrm{ps}$ pulse width. The excitation laser beam focuses on the sample through a $100 \times$ air objective lens $(\mathrm{NA}=0.95$, Olympus PLFLN 100X) with the spot radius of $0.624 \mu \mathrm{m}\left(1 / \mathrm{e}^{2}\right.$ of the maximum intensity measured with an EMCCD camera, DU-897U-CS0-\#BV, Andor, UK).

\section{RESULTS AND DISCUSSION}

Fig. 1a presents a schematic illustration of the memory device in the $\mathrm{Ag} / \mathrm{FA}_{0.83} \mathrm{MA}_{0.17} \mathrm{~Pb}\left(\mathrm{I}_{0.82} \mathrm{Br}_{0.18}\right)_{3} / \mathrm{FTO}$ configuration. Considering the cross-section view in Fig. 1b, a 

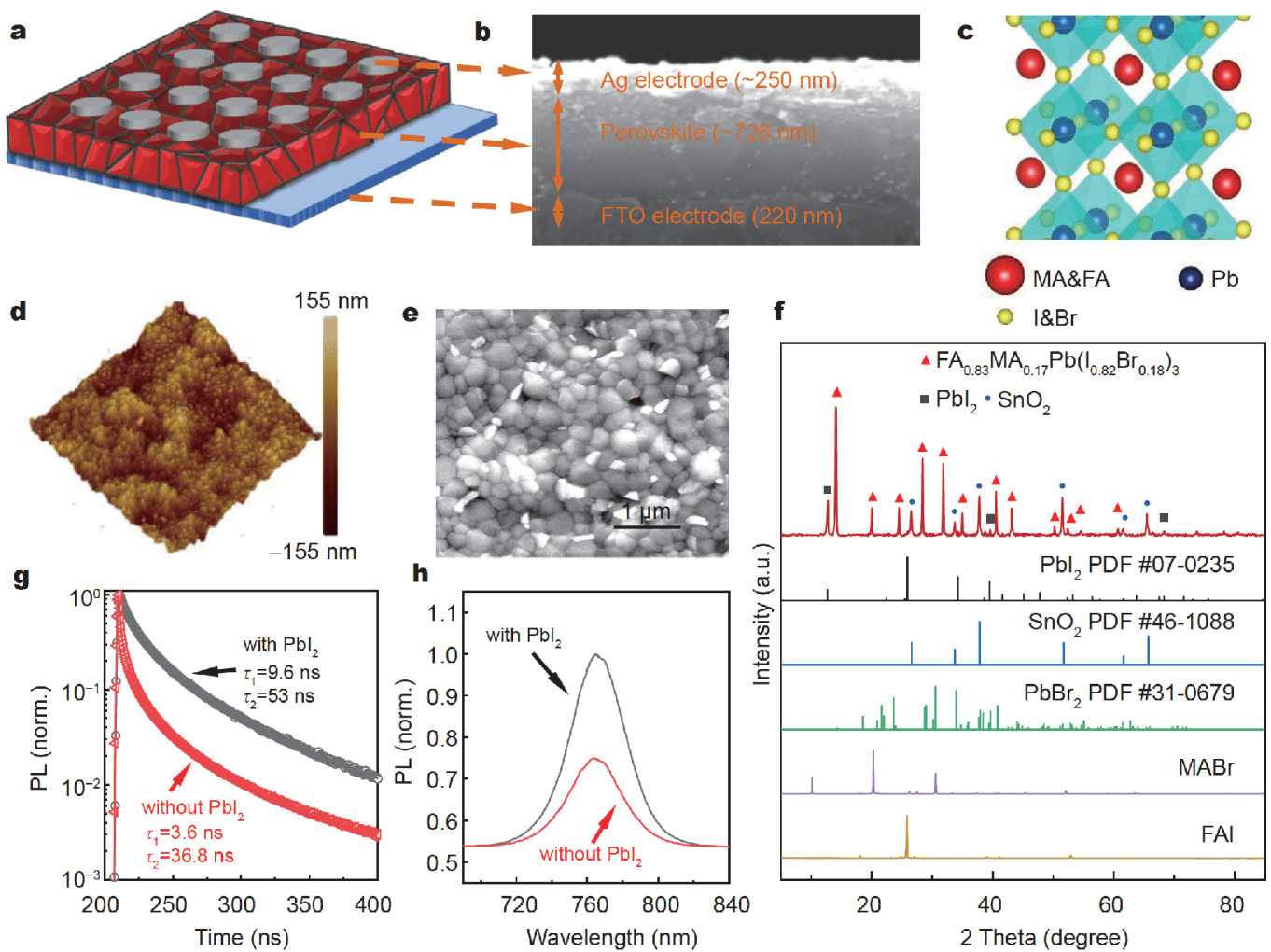

Figure 1 The device structure and film properties. (a) Illustration of the Ag/ $\mathrm{FA}_{0.83} \mathrm{MA}_{0.17} \mathrm{~Pb}\left(\mathrm{I}_{0.82} \mathrm{Br}_{0.18}\right)_{3} / \mathrm{FTO}$ device structure. (b) Cross-sectional SEM image of the device and the corresponding thickness. (c) Cubic crystal structure of the perovskite. (d) AFM image of the perovskite film (RMS = $38.2 \mathrm{~nm}$ ). (e) SEM image of the surface morphology. (f) XRD spectra of perovskite film of $\mathrm{FA}_{0.83} \mathrm{MA}_{0.17} \mathrm{~Pb}\left(\mathrm{I}_{0.82} \mathrm{Br}_{0.18}\right)_{3}$, precursors including FAI, MABr, $\mathrm{PbI}_{2}$ and $\mathrm{PbBr}_{2}$, and the substrate of FTO. (g) TRPL decay curves of perovskite films with $\mathrm{PbI}_{2}$ (black circle) and without $\mathrm{PbI}_{2}$ (red triangle) on glass/FTO substrate, excited with pulsed excitation $\left(406 \mathrm{~nm}, 500 \mathrm{kHz} \text { repetition rate, } 1 \mu \mathrm{W} \text { ). (h) PL measurement of the } \mathrm{FA}_{0.83} \mathrm{MA}_{0.17} \mathrm{~Pb} \mathrm{I}_{0.82} \mathrm{Br}_{0.18}\right)_{3}$ films with $\mathrm{PbI}_{2}$ (black line) and without $\mathrm{PbI}_{2}$ (red line) on glass/FTO substrate.

$\mathrm{FA}_{0.83} \mathrm{MA}_{0.17} \mathrm{~Pb}\left(\mathrm{I}_{0.82} \mathrm{Br}_{0.18}\right)_{3}$ thin film with $726 \mathrm{~nm}$ thickness was deposited onto an FTO electrode (thickness of $220 \mathrm{~nm}$ ) on a glass substrate using a one-step solution method. A $250 \mathrm{~nm}$-thick Ag layer was used as the top electrode, which was connected to an electric-switching source, and was deposited using mask-assisted thermal evaporation. Fig. 1c illustrates a simple crystal structure of the perovskite where $\mathrm{FA}^{+}$and $\mathrm{MA}^{+}$occupy the A sites and coordinate with the $\mathrm{PbX}_{6}$ octahedra, and the $\mathrm{I}^{-}$and $\mathrm{Br}^{-}$are located at the $\mathrm{X}$ sites. The surface roughness is as low as $38.2 \mathrm{~nm}$ as measured by AFM and shown in Fig. 1d. Therefore, the $\mathrm{FA}_{0.83} \mathrm{MA}_{0.17} \mathrm{~Pb}\left(\mathrm{I}_{0.82} \mathrm{Br}_{0.18}\right)_{3}$ layer would exhibit a low recombination rate. The grain boundaries, where the migrated ions induced by light/ electric fields can easily recombine, are reduced with the large particle size along with a smooth surface $[24,25]$. From the top-view surface morphology of the $\mathrm{FA}_{0.83^{-}}$ $\mathrm{MA}_{0.17} \mathrm{~Pb}\left(\mathrm{I}_{0.82} \mathrm{Br}_{0.18}\right)_{3}$ layer measured via SEM in Fig. 1e, the film is composed of crystalline particles. The particle size ranges from 120 to $476 \mathrm{~nm}$, which is larger than those in other studies with similar elemental compositions [24]. Additionally, a large number of brightly parasitic particles $(\sim 140 \mathrm{~nm})$ are distributed on the surface and grain boundaries of perovskite grains uniformly. The XRD pattern shows (Fig. 1f) that the diffraction peaks at $14.2^{\circ}, 19.9^{\circ}, 24.4^{\circ}, 28.4^{\circ}, 31.8^{\circ}, 34.9^{\circ}, 40.6^{\circ}, 43.2^{\circ}$, $50.3^{\circ}, 52.5^{\circ}, 54.6^{\circ}$ and $60.7^{\circ}$ are assigned to the cubic $\alpha$ perovskite crystal phase with high crystallinity. Meanwhile, a diffraction pattern of $\mathrm{PbI}_{2}$ at $12.7^{\circ}$ is observed [24,26-29]. These results demonstrate the formation of $\mathrm{PbI}_{2}$ which is similar to the results reported by others $[27,30]$. Additionally, the crystal phases of $\mathrm{PbBr}_{2}, \mathrm{FAI}$, and $\mathrm{MABr}$ are hardly detected except for some distinctive $\mathrm{SnO}_{2}$ peaks $\left(26.5^{\circ}, 33.8^{\circ}, 37.8^{\circ}, 51.7^{\circ}, 61.7^{\circ}, 65.7^{\circ}\right)$, which are from the FTO substrate. The TRPL decay kinetics (Fig. 1g) on a semilog plot is measured in $\mathrm{FA}_{0.83} \mathrm{MA}_{0.17} \mathrm{~Pb}\left(\mathrm{I}_{0.82} \mathrm{Br}_{0.18}\right)_{3}$ perovskite. The fast decay component, $\tau_{1}$, might come from bimolecular recombination [31]. The long decay component, $\tau_{2}$, could be attributed to recombination of free carriers in the ra- 
diative channel [1]. The $\tau_{1}$ and $\tau_{2}$ of the sample with $\mathrm{PbI}_{2}$ are longer than that without $\mathrm{PbI}_{2}$. Meanwhile, the PL measurement gives a corresponding result in Fig. 1h, and the excitation intensity in the film with $\mathrm{PbI}_{2}$ is enhanced compared with the film without $\mathrm{PbI}_{2}$. This demonstrates that a moderate amount of $\mathrm{PbI}_{2}$ has a positive effect due to effective passivation of surface/grain boundary defects.

Fig. 2a presents the analog switching characteristic of the $\mathrm{Ag} / \mathrm{FA}_{0.83} \mathrm{MA}_{0.17} \mathrm{~Pb}\left(\mathrm{I}_{0.82} \mathrm{Br}_{0.18}\right)_{3} /$ FTO device with a voltage below $0.5 \mathrm{~V}$. A DC voltage was applied on the Ag top electrode while the bottom FTO electrode was grounded. A compliance current of $1 \mathrm{~mA}$ was set to prevent thermal-induced breakdown. When six consecutive positive voltage sweeps of $0 \rightarrow+0.5 \rightarrow 0 \mathrm{~V}$ were applied to the device, the current amplitude gradually increased after each sweep. By contrast, when six consecutive negative voltage sweeps of $0 \rightarrow-0.5 \rightarrow 0 \mathrm{~V}$ were applied to the device, the current amplitude gradually decreased after each sweep. Fig. $2 \mathrm{~b}$ shows the direct changes in the conductance with continuous voltage sweeps.

To more clearly demonstrate the induced current change from consecutive voltage sweeps, current responses with continuously applied pulses were measured and shown in Fig. $2 \mathrm{c}$ and d. Twenty sequential positive and negative pulses $(+0.6 \mathrm{~V} /-0.5 \mathrm{~V}$ at $2 \mathrm{~ms})$ were applied to the Ag electrodes, and the current either increased or decreased as the number of pulses increased. These results illustrate that the conductance is also gradually potentiated or depressed by successive pulses.

Interestingly, when the SET voltage was raised to $+0.8 \mathrm{~V}$, the device exhibited threshold switching characteristics without a forming process, as shown in Fig. 2e. As a positive voltage sweep was applied from 0 to $+0.8 \mathrm{~V}$, the device was switched from a high resistance state (HRS) to a low resistance state (LRS) at $+0.65 \mathrm{~V}$. Then, the device returned to the HRS in a voltage sweep from 0 to $-1.5 \mathrm{~V}$. Fig. $2 \mathrm{f}$ demonstrates the endurance test for 40 cycles, and the HRS/LRS ratio of the perovskite memory cell is greater than 10. It should be noted that all of the above electrical tests were performed under dark conditions.

As mentioned above, when the SET voltage was $0.5 \mathrm{~V}$, several sweeps were needed to reach the LRS. However, when the voltage increased to $0.8 \mathrm{~V}$, only one stimulus was sufficient to reach LRS. In other words, increasing the bias amplitude reduces the number of required stimuli, and the device requires only one stimulus to transit from HRS to LRS. Thus, an appropriate bias voltage could achieve the transition from an analog switch to a threshold switch.

Based on the device structure and composition, a
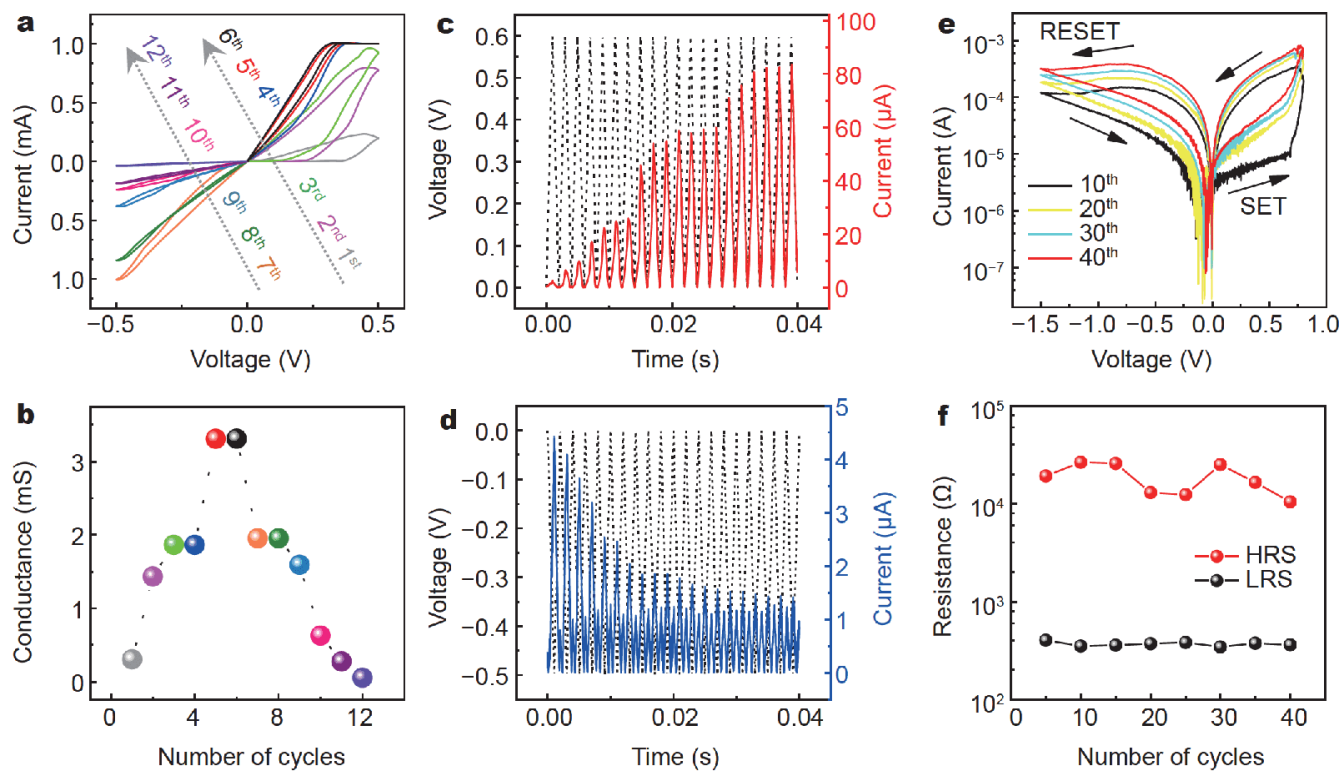

Figure 2 The analog switching (a-d) and threshold switching (e, f) characteristics for the Ag/ $\mathrm{FA}_{0.83} \mathrm{MA}_{0.17} \mathrm{~Pb}_{0.82} \mathrm{I}_{0.18} \mathrm{Br}_{0} / \mathrm{FTO}$. (a) $I-V$ characteristics of the device during six consecutive positive $(0 \rightarrow+0.5 \rightarrow 0 \mathrm{~V})$ and negative $(0 \rightarrow-0.5 \rightarrow 0 \mathrm{~V})$ sweeps. (b) Changes of the device conductance during consecutive sweeps. (c) Current responses with time based on the positive pulses $(+0.6 \mathrm{~V}$ at $2 \mathrm{~ms})$. (d) Current responses with time based on the negative pulses $(-0.5 \mathrm{~V}$ at $2 \mathrm{~ms})$. (e) $I-V$ characteristics of the device from four series sweeps $(0 \rightarrow+0.8 \rightarrow 0 \rightarrow-1.5 \rightarrow 0 \mathrm{~V})$. (f) Endurance performance read at $0.05 \mathrm{~V}$. 
proposed mechanism for the resistive switching behavior may be derived from two primary reasons. Firstly, the $I-$ $V$ switching behavior under electric fields could arise from the nature of this OHP material including the ionic migration and charge trapping/detrapping effects related with defect states inside the perovskite films [32]. In our work, as mentioned above, the defect sites derived from the surface or grain particles of the OHPs have been passivated by the excess of crystalline $\mathrm{PbI}_{2}$ during the fabrication process. Therefore it is necessary to pay more attention to the ionic migration which has been widely reported due to its lower activation energy $[13,33]$. Ionic migration is assumed to be one of the possible reasons for the switching behavior in these memory devices. The accumulation of ions resulting from ionic migration induced by external electric fields could explain the variations in the current intensities. Based on the $\mathrm{FA}_{0.83} \mathrm{MA}_{0.17} \mathrm{~Pb}\left(\mathrm{I}_{0.82} \mathrm{Br}_{0.18}\right)_{3}$ material, the migration candidates include $\mathrm{MA}^{+}, \mathrm{FA}^{+}, \mathrm{I}^{-}$, and $\mathrm{Br}^{-}$ions. Many researchers have suggested that the migration of $\mathrm{I}^{-}$ions is dominant due to the lowest activation energy $[34,35]$ and high diffusion coefficient [10]. Secondly, the Ag electrode can easily diffuse to form a metal filament, especially after the setting process [36]. Therefore, the Ag conductive filament is assumed to be the other alternative candidate reason to the switching behavior in the memory device.

To further verify the hypotheses, the switching behavior was first studied using the measurement device, which has another architecture $\left(\mathrm{Au} / \mathrm{FA}_{0.83} \mathrm{MA}_{0.17} \mathrm{~Pb}\left(\mathrm{I}_{0.82} \mathrm{Br}_{0.18}\right)_{3} /\right.$ FTO), as demonstrated in Fig. 3a, where the top Ag electrode is replaced with $\mathrm{Au}$. In the $I-V$ curves from Fig. $3 \mathrm{~b}$ and $\mathrm{c}$, subjected to positive voltage $(0 \rightarrow 1.5 \rightarrow 0 \mathrm{~V})$ sweeps with five cycles, the device presents a variety of variable $I-V$ hysteresis loops with increasing voltage sweep cycles, where the current intensity in each cycle at the same potential gradually decreases. From these results, the electrical characteristics based on the $\mathrm{FA}_{0.83} \mathrm{MA}_{0.17} \mathrm{~Pb}\left(\mathrm{I}_{0.82} \mathrm{Br}_{0.18}\right)_{3}$ arise from ions migration and accumulation. After applying an external electric field, the $\mathrm{I}^{-}$ions transfer towards the opposite electric direction [37]. In the case of high-energy stimulated situation with a strong voltage, a small amount of $\mathrm{Br}^{-}$ions accompanied with some $\mathrm{MA}^{+}$and $\mathrm{FA}^{+}$could also actively migrate and move along the positive and negative directions of the electric field, respectively [38]. Then, a built-in field, which is opposite to the external electric field, is generated due to the migrating charge accumulation. This field can dramatically decrease the effective potential that is applied to the measured device. Therefore, the current intensity is significantly weakened compared with the first and second voltage circles shown in Fig. 3b. When increasing the voltage-scan circles or extending the electric-field excitation time, the enhanced charge accumulation leads to an increased built-in electric field. Consequently, the current intensity gradually decreases as the effective potential decreases along with the increasing voltage scan circles. This is further verified by the negative voltage scan circles shown in Fig. 3c. A more intuitive schematic of the above phenomena is demonstrated in Fig. 3d.

In addition, SEM accompanied with EDS was used to characterize the ionic migration under electric fields and the formation of an Ag metal filament in the fabricated memory devices. Fig. $4 \mathrm{a}$ and $\mathrm{c}$ show the EDS profiles of the cross-sectional elemental distribution before and after applying a positive voltage stress to the Ag electrode, and Fig. $4 \mathrm{~b}$ shows the corresponding cross-sectional SEM image of the device. For the EDS profile (Fig. 4a) without applying a voltage stress, the content of $I$ is consistent with that of $\mathrm{Pb}$, which gradually decreases towards the electrode. This means that I atoms are stable within the perovskite before the applied voltage. The Ag, as an active metal, can be easily oxidized into $\mathrm{Ag}^{+}$after exposure to ambient air and react with the halide ions in the perovskite [37]. After applying a positive voltage stress onto the Ag electrode, Fig. 4c shows the I is increasingly concentrated at the upper perovskite layer and supports
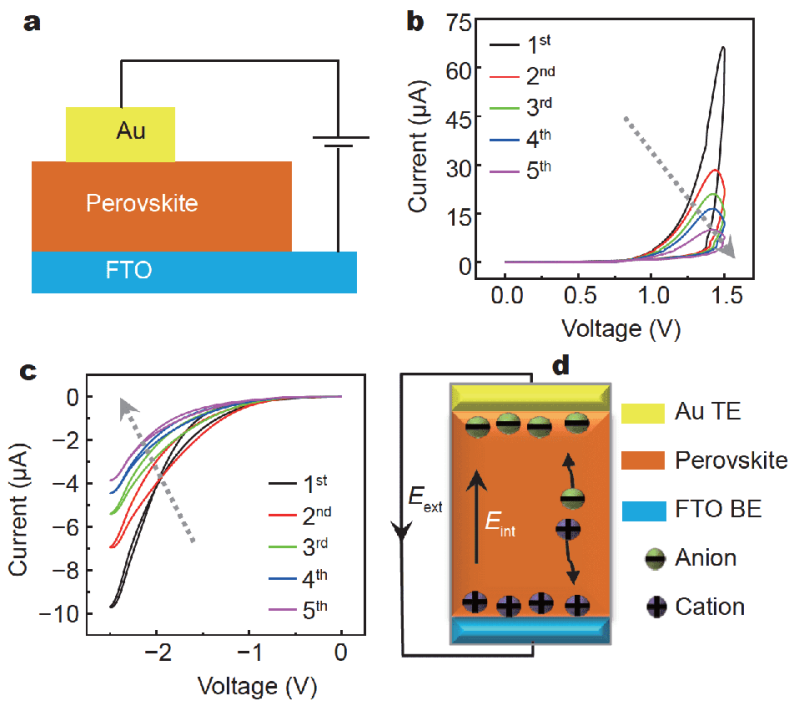

Figure 3 (a) Illustration of the structure and characterization schematics of the $\mathrm{Au} / \mathrm{FA}_{0.83} \mathrm{MA}_{0.17} \mathrm{~Pb}\left(\mathrm{I}_{0.82} \mathrm{Br}_{0.18}\right)_{3} / \mathrm{FTO}$ device. (b) $I-V$ characteristics of the device during six consecutive positive $(0 \rightarrow+1.5 \rightarrow 0 \mathrm{~V})$ sweeps. (c) $I-V$ characteristics of the device during six consecutive negative $(0 \rightarrow-2.5 \rightarrow 0 \mathrm{~V})$ sweeps. (d) The formation process of the built-in electric field under the action of an external electric field. 

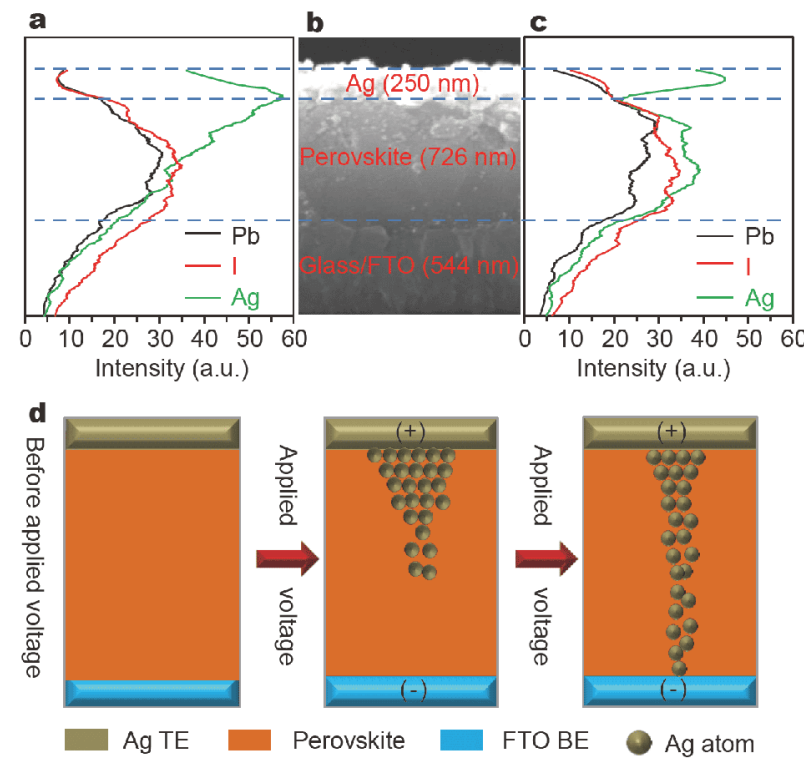

Figure 4 EDS analysis of the $\mathrm{Ag} / \mathrm{FA}_{0.83} \mathrm{MA}_{0.17} \mathrm{~Pb}\left(\mathrm{I}_{0.82} \mathrm{Br}_{0.18}\right)_{3} / \mathrm{FTO}$ device and the formation of the $\mathrm{Ag}$ conductive filament. (a) EDS profiles of the cross-sectional element distribution before applying a positive voltage to the Ag electrode. (b) The cross-sectional SEM image corresponding to the EDS profiles. (c) EDS profiles of the cross-sectional element distribution after applying a positive voltage on the Ag electrode. (d) The formation process of the $\mathrm{Ag}$ conductive filament with a positive voltage on the Ag electrode.

that the $\mathrm{I}^{-}$ions migrate towards the opposite direction of the electric field [10].

For the Ag element, the atomic content significantly increased towards the cathode and decreased dramatically at the interface between the $\mathrm{Ag}$ electrode and the perovskite layer. This means that atomic Ag migration occurred under an applied electric field. As a result, the resistance decreased due to a formed Ag filament that bridged both electrodes. Fig. 4d exhibits the formation process of the conductive filament with a positive voltage on the Ag electrode. Meanwhile, it has been previously explained that the Ag migration can be ascribed to thermal driven diffusion and bipolar electrode effects $[39,40]$. Under a voltage stress, the current passes throughout the entire resistance switching layer and heats some regions, which provides a thermal channel for Ag migration towards the cathode with the aid of the bipolar electrodes. At the same time, oxidation reactions occur in the interface between the Ag electrode and the perovskite layer [41,42]. After oxidation, the $\mathrm{Ag}^{+}$migrates through the perovskite layer and is again reduced into atomic $\mathrm{Ag}$ with the electrons from the cathode. This explains why the Ag content at the interface region decreased dramatically after the voltage was applied.
However, in the Ag-based device, it is still unclear whether the intrinsic properties of the OHPs or the $\mathrm{Ag}$ dominate regarding the switching behavior. It is noteworthy that the electrical characteristics of the Ag-based device in Fig. 2a are different from that of the Au-based device in Fig. $3 \mathrm{~b}$ and $\mathrm{c}$.

Firstly, the conductance of the Ag-based device gradually grew with a repeated forward scan voltage, while the conductance of the Au-based device gradually reduced. Secondly, in the same scan interval $(0$ to $+0.5 \mathrm{~V})$, the current intensity of the Ag-based device was larger than that for the Au-based device.

As the previous analysis, ionic migration and the resulting built-in potential field contribute to the $I-V$ switching characteristics in the Au-based device. $\mathrm{The}^{+} \mathrm{gg}^{+}$ ions dominate the resistive switching properties of the Ag-based device, since Ag is easily oxidized by an electric field and migrates in the field direction. When a positive bias was applied to the Ag electrode, Ag atoms could be dissolved to $\mathrm{Ag}^{+}$ions, which subsequently diffused towards the bottom FTO electrode and were reduced to atomic Ag, in the switching layer. Thus, the Ag atoms gradually piled-up between the Ag and FTO electrodes and $\mathrm{Ag}$ conducting filaments were grown from the reduced Ag atoms, leading to a narrowed electrode separation. Therefore, when a periodic positive voltage was applied to the Ag electrode, the gap between the top and the bottom electrodes decreased and the lateral width of the conducting filaments increased, which resulted in the increasing current (Fig. 2c). By contrast, with a successive negative bias, the Ag conducting filaments gradually decreased due to the Ag oxidation, which led to the decreasing current (Fig. 2d).

In Fig. $2 \mathrm{a}-\mathrm{d}$, when the SET voltage was $0.5 \mathrm{~V}$, the device conductance continuously changed with the cyclic stimuli. This change was related to both the amount of stimulations and the stimulus frequency $[43,44]$. Next, frequency-dependent tests (Fig. 5a) illustrate spike-ratedependent plasticity (SRDP) $[16,45]$, one of the basic characteristics of synaptic plasticity. To investigate the influence of the spiking rate, we used pairs of consecutive pulses separated by different intervals, where each stimulation cycle was one pulse $(0.5 \mathrm{~V}$ at $1 \mathrm{~ms})$. Fig. $5 \mathrm{a}$ demonstrates the relationship between the weight change $(\Delta W)$ and the pulse interval with successive pulses. The weight change is given by $\Delta W=\left(G_{n}-G_{1}\right) / G_{1}$, where $\Delta G$ is the change in conductance between two pulses, and $G_{1}$ and $G_{n}$ are the initial and $n$-th pulse conductance, respectively.

When the stimulation interval was short $(10 \mu \mathrm{s})$, the 

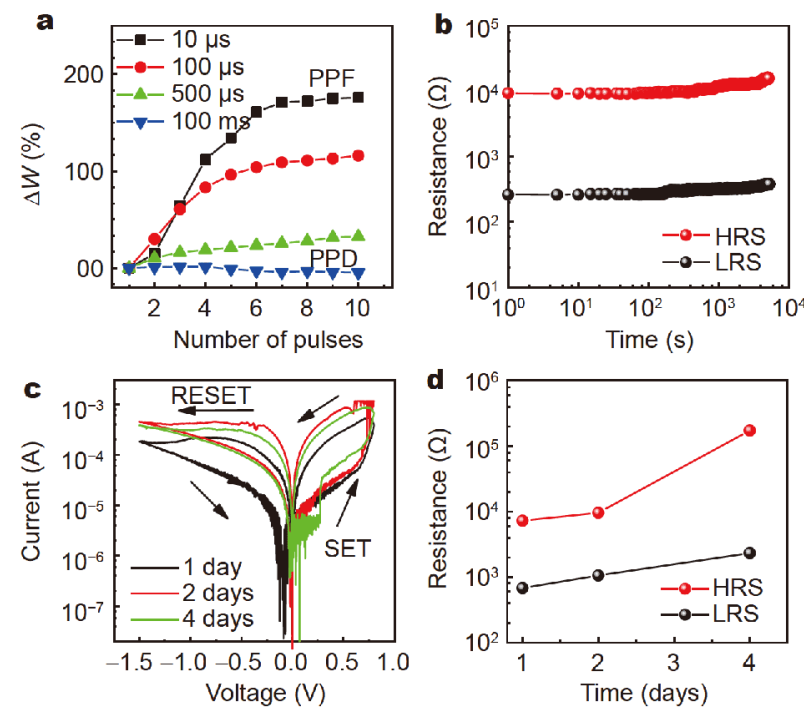

Figure 5 The short-term plasticity of the synapse and stability of the $\mathrm{Ag} / \mathrm{FA}_{0.83} \mathrm{MA}_{0.17} \mathrm{~Pb}\left(\mathrm{I}_{0.82} \mathrm{Br}_{0.18}\right)_{3} / \mathrm{FTO}$ device. (a) The change in the conductance (weight) percentage given by different pulse intervals. When the pulse interval increases from $10 \mu \mathrm{s}$ to $100 \mathrm{~ms}$, the weight change reduces with the rise of pulse number and finally reverses. (b) Retention behaviors of the device. (c) $I-V$ characteristics of the device over 3 days. (d) The fluctuation in HRS and LRS corresponding to (c).

weight change increased significantly with an increase in the number of pulses, which was similar to the pairedpulse facilitation (PPF) [46] in bio-synapses. As the stimulation interval increased, the increase in the weight change became less pronounced. In fact, the weight change began to decrease as the stimulation interval increased to $100 \mathrm{~ms}$, which was similar to the paired-pulse depression (PPD) in bio-synapses [46].

In bio-synapses, stimulation from the pre-synapses triggers the release of calcium ions. Their concentration affects the release of neurotransmitters, which amplifies the ability of synapses to transmit signals. However, the concentration of calcium ions gradually returns to its initial value over time. When the interval between two stimulations is less than the time when the concentration of calcium ions returns to the initial state, the response of the post-synapses is stronger than the previous one, which is called PPF. Similarly, if the interval between the two stimulations is more than the time, the calcium ions channels are partially inactivated before the second stimulation comes. In this case, the calcium ions concentration is lower than the previous value, and therefore the response of the post-synapses is weaker than the previous one, which is called PPD [47]. The above experimental results demonstrate that the memory device shows a functionality that is analogous to artificial synapses. Thus, when a pulse train with varying intervals is applied, the memory device exhibits different biosynaptic characteristics (PPF and PPD), which is consistent with the current research on PPF and PPD memristor artificial synapses [48,49].

The above observations may be due to the accumulation of $\mathrm{Ag}^{+}$in the perovskite films, which leads to the gradual growth of Ag conductive filaments between the two electrodes during the short intervals of continuous stimulations. When a pulse train with long intervals is applied, the facilitation turns into depression, because the strength of the applied electric field is weakened with longer pulse intervals and the spontaneous diffusion effects of the Ag atoms are dominant [48]. By observing the dependence of the weight change and stimulation frequency, we were able to simulate the synaptic short-term plasticity from PPF to PPD.

When the SET voltage was $0.8 \mathrm{~V}$, the device exhibited a threshold switching characteristic, as given in Fig. 2e, under a DC voltage bias sweep. To verify the stability of the HRS and LRS, the retention of each state was measured at a reading voltage of $0.05 \mathrm{~V}$ for $5.2 \times 10^{3} \mathrm{~s}$, as shown in Fig. 5b. In this case, the memory device exhibited good non-volatile characteristics. We further characterized the electrical properties to demonstrate the stability in Fig. $5 c$ and d. The inverse hysteresis window of the curve $(0 \rightarrow-1.5 \rightarrow 0 \mathrm{~V})$ tested on the fourth day became smaller, while both the HRS and the LRS became larger. A window of 10 times (HRS/LRS) indicated the stability of the device.

\section{CONCLUSION}

We demonstrated a device that exhibits resistive behaviors based on the material structure $\mathrm{Ag} / \mathrm{FA}_{0.83} \mathrm{MA}_{0.17} \mathrm{~Pb}$ $\left(\mathrm{I}_{0.82} \mathrm{Br}_{0.18}\right)_{3} / \mathrm{FTO}$. The conductive Ag filaments play a decisive role in the resistive behavior. By controlling the appropriate SET voltage, two kinds of resistance characteristics of an analog switch and threshold switch can be realized. When the SET voltage is low, the analog switching characteristics can be realized through the successive growth and contraction of the conductive Ag filaments. The device conductance continuously changes with consecutive voltage pulses and mimics the shortterm plasticity of the synapses (PPF and PPD), which can be useful in the future neuromorphic computing devices. When the SET voltage is high, we realized the threshold switching characteristics through the formation and fracturing of the Ag conductive filaments. The conductance was abrupt with the applied voltage and had a suitable switch window and hold time. Thus, the pro- 
posed device can be a promising candidate for non-volatile memory applications.

\section{Received 10 February 2019; accepted 23 April 2019; published online 17 May 2019}

1 Ponseca Jr. CS, Savenije TJ, Abdellah M, et al. Organometal halide perovskite solar cell materials rationalized: ultrafast charge generation, high and microsecond-long balanced mobilities, and slow recombination. J Am Chem Soc, 2014, 136: 5189-5192

2 Yin WJ, Shi T, Yan Y. Unique properties of halide perovskites as possible origins of the superior solar cell performance. Adv Mater, 2014, 26: 4653-4658

3 Shi D, Adinolfi V, Comin R, et al. Low trap-state density and long carrier diffusion in organolead trihalide perovskite single crystals. Science, 2015, 347: 519-522

4 Wu X, Trinh MT, Niesner D, et al. Trap states in lead iodide perovskites. J Am Chem Soc, 2015, 137: 2089-2096

5 Miyata A, Mitioglu A, Plochocka P, et al. Direct measurement of the exciton binding energy and effective masses for charge carriers in organic-inorganic tri-halide perovskites. Nat Phys, 2015, 11: $582-587$

6 Correa-Baena JP, Saliba M, Buonassisi T, et al. Promises and challenges of perovskite solar cells. Science, 2017, 358: 739-744

7 Cho $\mathrm{H}$, Jeong SH, Park $\mathrm{MH}$, et al. Overcoming the electroluminescence efficiency limitations of perovskite light-emitting diodes. Science, 2015, 350: 1222-1225

8 Su L, Zhao ZX, Li HY, et al. High-performance organolead halide perovskite-based self-powered triboelectric photodetector. ACS Nano, 2015, 9: 11310-11316

9 Su L, Zhao ZX, Li HY, et al. Photoinduced enhancement of a triboelectric nanogenerator based on an organolead halide perovskite. J Mater Chem C, 2016, 4: 10395-10399

10 Eames C, Frost JM, Barnes PRF, et al. Ionic transport in hybrid lead iodide perovskite solar cells. Nat Commun, 2015, 6: 7497

11 Yuan Y, Huang J. Ion migration in organometal trihalide perovskite and its impact on photovoltaic efficiency and stability. Acc Chem Res, 2016, 49: 286-293

$12 \mathrm{Wu} \mathrm{T}$, Mukherjee R, Ovchinnikova OS, et al. Metal/ion interactions induced $\mathrm{p}-\mathrm{i}-\mathrm{n}$ junction in methylammonium lead triiodide perovskite single crystals. J Am Chem Soc, 2017, 139: 17285-17288

13 Li C, Guerrero A, Zhong Y, et al. Origins and mechanisms of hysteresis in organometal halide perovskites. J Phys-Condens Matter, 2017, 29: 193001

14 Yoo EJ, Lyu M, Yun JH, et al. Resistive switching behavior in organic-inorganic hybrid $\mathrm{CH}_{3} \mathrm{NH}_{3} \mathrm{PbI}_{3-x} \mathrm{Cl}_{x}$ perovskite for resistive random access memory devices. Adv Mater, 2015, 27: 6170-6175

15 Stoumpos CC, Malliakas CD, Kanatzidis MG. Semiconducting tin and lead iodide perovskites with organic cations: Phase transitions, high mobilities, and near-infrared photoluminescent properties. Inorg Chem, 2013, 52: 9019-9038

16 Xiao Z, Huang J. Energy-efficient hybrid perovskite memristors and synaptic devices. Adv Electron Mater, 2016, 2: 1600100

$17 \mathrm{Xu} \mathrm{W}$, Cho H, Kim YH, et al. Organometal halide perovskite artificial synapses. Adv Mater, 2016, 28: 5916-5922

18 Leijtens $\mathrm{T}$, Bush $\mathrm{K}$, Cheacharoen $\mathrm{R}$, et al. Towards enabling stable lead halide perovskite solar cells; interplay between structural, environmental, and thermal stability. J Mater Chem A, 2017, 5: $11483-11500$
19 Rehman W, McMeekin DP, Patel JB, et al. Photovoltaic mixedcation lead mixed-halide perovskites: links between crystallinity, photo-stability and electronic properties. Energy Environ Sci, 2017, 10: $361-369$

20 Xie LQ, Chen L, Nan ZA, et al. Understanding the cubic phase stabilization and crystallization kinetics in mixed cations and halides perovskite single crystals. J Am Chem Soc, 2017, 139: 33203323

21 Chen J, Xu J, Xiao L, et al. Mixed-organic-cation (FA) $)_{x}(\mathrm{MA})_{1-x} \mathrm{PbI}_{3}$ planar perovskite solar cells with $16.48 \%$ efficiency via a lowpressure vapor-assisted solution process. ACS Appl Mater Interfaces, 2017, 9: 2449-2458

22 Isikgor $\mathrm{FH}, \mathrm{Li} \mathrm{B}$, Zhu $\mathrm{H}$, et al. High performance planar perovskite solar cells with a perovskite of mixed organic cations and mixed halides, $\mathrm{MA}_{1-x} \mathrm{FA}_{x} \mathrm{PbI}_{3-y} \mathrm{Cl}_{y}$. J Mater Chem A, 2016, 4: $12543-$ 12553

23 Zhang Y, Grancini G, Feng Y, et al. Optimization of stable quasicubic $\mathrm{FA}_{x} \mathrm{MA}_{1-x} \mathrm{PbI}_{3}$ perovskite structure for solar cells with efficiency beyond 20\%. ACS Energy Lett, 2017, 2: 802-806

24 Wang $\mathrm{Y}, \mathrm{Wu}$ J, Zhang $\mathrm{P}$, et al. Stitching triple cation perovskite by a mixed anti-solvent process for high performance perovskite solar cells. Nano Energy, 2017, 39: 616-625

25 Zhao $\mathrm{Z}$, Chen $\mathrm{X}, \mathrm{Wu} \mathrm{H}$, et al. Probing the photovoltage and photocurrent in perovskite solar cells with nanoscale resolution. Adv Funct Mater, 2016, 26: 3048-3058

26 Saliba M, Matsui T, Seo JY, et al. Cesium-containing triple cation perovskite solar cells: improved stability, reproducibility and high efficiency. Energy Environ Sci, 2016, 9: 1989-1997

27 Wang S, Zhang C, Feng Y, et al. High-air-flow-velocity assisted intermediate phase engineering for controlled crystallization of mixed perovskite in high efficiency photovoltaics. J Mater Chem A, 2018, 6: 8860-8867

28 Brunetti B, Cavallo C, Ciccioli A, et al. On the thermal and thermodynamic (in)stability of methylammonium lead halide perovskites. Sci Rep, 2016, 6: 31896

29 Jung $\mathrm{K}$, Lee $\mathrm{JH}, \mathrm{Oh} \mathrm{K}$, et al. Efficient composition tuning via cation exchange and improved reproducibility of photovoltaic performance in $\mathrm{FA}_{x} \mathrm{MA}_{1-x} \mathrm{PbI}_{3}$ planar heterojunction solar cells fabricated by a two-step dynamic spin-coating process. Nano Energy, 2018, 54: 251-263

30 Chen Q, Zhou H, Song TB, et al. Controllable self-induced passivation of hybrid lead iodide perovskites toward high performance solar cells. Nano Lett, 2014, 14: 4158-4163

31 Gunawan O, Todorov TK, Mitzi DB. Loss mechanisms in hydrazine-processed $\mathrm{Cu}_{2} \mathrm{ZnSn}(\mathrm{Se}, \mathrm{S})_{4}$ solar cells. Appl Phys Lett, 2010, 97: 233506

32 van Reenen S, Kemerink M, Snaith HJ. Modeling anomalous hysteresis in perovskite solar cells. J Phys Chem Lett, 2015, 6: 3808-3814

33 Richardson G, O'Kane SEJ, Niemann RG, et al. Can slow-moving ions explain hysteresis in the current-voltage curves of perovskite solar cells? Energy Environ Sci, 2016, 9: 1476-1485

34 Jaysankar M, Qiu W, Bastos J, et al. Crystallisation dynamics in wide-bandgap perovskite films. J Mater Chem A, 2016, 4: 1052410531

35 Li C, Tscheuschner S, Paulus F, et al. Iodine migration and its effect on hysteresis in perovskite solar cells. Adv Mater, 2016, 28 : 2446-2454

36 Wang Y, Lv Z, Liao Q, et al. Synergies of electrochemical metallization and valance change in all-inorganic perovskite quantum 
dots for resistive switching. Adv Mater, 2018, 30: 1800327

37 Zhu X, Lee J, Lu WD. Iodine vacancy redistribution in organicinorganic halide perovskite films and resistive switching effects. Adv Mater, 2017, 29: 1700527

38 Yuan Y, Chae J, Shao Y, et al. Photovoltaic switching mechanism in lateral structure hybrid perovskite solar cells. Adv Energy Mater, 2015, 5: 1500615

39 Midya R, Wang Z, Zhang J, et al. Anatomy of Ag/hafnia-based selectors with $10^{10}$ nonlinearity. Adv Mater, 2017, 29: 1604457

40 Yang Y, Gao P, Li L, et al. Electrochemical dynamics of nanoscale metallic inclusions in dielectrics. Nat Commun, 2014, 5: 4232

41 Li Y, Long S, Liu Q, et al. Resistive switching performance improvement via modulating nanoscale conductive filament, involving the application of two-dimensional layered materials. Small, 2017, 13: 1604306

42 Chen B, Shi J, Zheng X, et al. Ferroelectric solar cells based on inorganic-organic hybrid perovskites. J Mater Chem A, 2015, 3: 7699-7705

43 Park Y, Lee JS. Artificial synapses with short- and long-term memory for spiking neural networks based on renewable materials. ACS Nano, 2017, 11: 8962-8969

44 Wang Z, Joshi S, Savel'ev SE, et al. Memristors with diffusive dynamics as synaptic emulators for neuromorphic computing. Nat Mater, 2017, 16: 101-108

45 Li Y, Zhong Y, Zhang J, et al. Activity-dependent synaptic plasticity of a chalcogenide electronic synapse for neuromorphic systems. Sci Rep, 2014, 4: 4906

46 Zucker RS, Regehr WG. Short-term synaptic plasticity. Annu Rev Physiol, 2002, 64: 355-405

47 CG Galizia, P-M Lledo. Neurosciences-From Molecule To Behavior: A University Textbook. Berlin: Springer, 2013

48 Yan X, Zhou Z, Zhao J, et al. Flexible memristors as electronic synapses for neuro-inspired computation based on scotch tapeexfoliated mica substrates. Nano Res, 2018, 11: 1183-1192

49 Wang Z, Midya R, Joshi S, et al. Unconventional computing with diffusive memristors. In: 2018 IEEE International Symposium on Circuits and Systems (ISCAS). 2018, 1-5

Acknowledgements We acknowledge the financial supports from the National Natural Science Foundation of China (51872036, 51773025), Dalian Science and Technology Innovation Fund (2018J12GX033), National Key R\&D Program of China (2017YFB0405604)

Author contributions Huang $\mathrm{Y}$ and Zhao Z, Wang $\mathrm{C}$ and $\mathrm{Wu} \mathrm{H}$ designed the experiment. Huang $\mathrm{Y}$ prepared the perovskite precursor solution, characterized the SEM, AFM, XRD, EDS of all the films and electrical features of all devices. Fan $\mathrm{H}$ prepared the perovskite films and participated in the characterizations. Huang $\mathrm{Y}$, Wang $\mathrm{C}$ and Zhao Z prepared the manuscript. Bian J, Yang $\mathrm{Y}$ and $\mathrm{Wu} \mathrm{H}$ helped revise the manuscript. The final version of the manuscript was approved by all authors.

Conflict of interest The authors declare no conflict of interest.

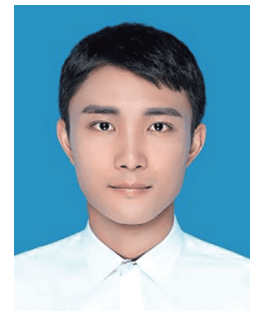

Yang Huang received the BSc degree in electronic science and technology from Hebei University of Science and Technology in 2014. Currently, he is a $\mathrm{PhD}$ candidate in Dalian University of Technology under the supervision of Prof. Jiming Bian and Prof. Chen Wang. His current research focuses on the structure design and working mechanism of the resistive random access memory (RRAM) and synaptic bionics based on perovskite material.

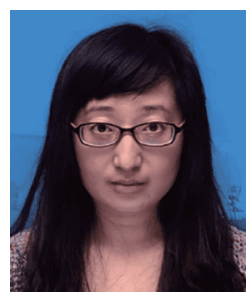

Zhenxuan Zhao received her $\mathrm{PhD}$ degree in chemical material engineering from Beijing University of Technology in 2013 and finished her post-doctor job in Beijing Institute of $\mathrm{Na}$ noenergy and Nanosystems, Chinese Academy of Sciences in 2015. Currently, her research interest focuses on nanomaterials and devices in Institute of Microelectronics, Tsinghua University.

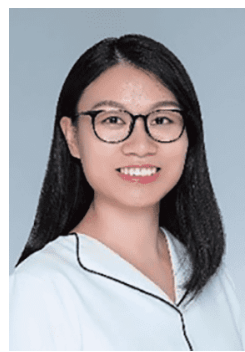

Chen Wang received her Joint- $\mathrm{PhD}$ degree (2012) in microelectronics and solid-state electronics from Fudan University (China) and Purdue University (USA). She is now an associate professor and $\mathrm{PhD}$ supervisor in the School of Microelectronics, Dalian University of Technology. Her research covers topics ranging from basic material physics to scalable manufacturing of electronic/photonic/photovoltaic devices.

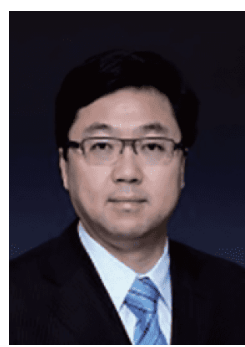

Huaqiang $\mathrm{Wu}$ received his $\mathrm{PhD}$ degree in electrical engineering from Cornell University, Ithaca, NY, in 2005. Currently, he is the deputy director of the Institute of Microelectronics, Tsinghua University, Beijing, China and the director of Micro/Nano Fabrication Center and deputy director of Beijing Innovation Center for Future Chips. His research interest includes emerging memory and neuromorphic computing technologies.

\section{电导金属细丝主导的杂化钙铁矿基存储器}

黄阳 ${ }^{1,3}$, 赵振璇 ${ }^{2 *}$, 王晨 ${ }^{1 *}$, 㚞宏波 ${ }^{1}$ 杨一鸣 ${ }^{1}$, 边继明 ${ }^{3}$, 吴华强 ${ }^{2 *}$

摘要 有机-无机杂化钙钛矿 (OHPs) 作为太阳能电池中的光吸收材 料备受重视, 并且在电阻开关 (RS) 存储器的应用中引起了广泛关 注. 以前的研究表明, 在外电场作用下钻钛矿中的离子能够发生迁 移并形成导电通道. 然而, 主导其阻变行为的是 $A g$ 还是卤素仍然存 在着争议. 本文中, 我们研究了一种基于 $\mathrm{Ag} / \mathrm{FA}_{0.83} \mathrm{MA}_{0.17} \mathrm{~Pb}$ $\left(\mathrm{I}_{0.82} \mathrm{Br}_{0.18}\right)_{3} / \mathrm{FTO}$ (掺氟的氧化锡) 的电阻开关存储器. 在开启过程 (在 $\mathrm{Ag}$ 电极端施加正向扫描电压) 完成后, 我们通过EDS (能量色散 $\mathrm{X}$ 射线谱)发现了银离子和卤素离子的迁移. 并通过对比基于 $\mathrm{Au}$ 电 极器件的电流-电压特征曲线, 发现由 $\mathrm{Ag}$ 形成的导电通道是影响 $\mathrm{Ag}$ 基器件开关特性的主要因素. 同时, 通过控制合适大小的开启电压, 基于 $\mathrm{Ag}$ 电极的电阻开关器件实现了模拟开关和阈值开关两种不同 的阻变开关特性. 因此, 在未来有可能在单个器件中同时实现数据 存储和神经形态计算两种功能. 